\title{
Effects of MDP-based primers on shear bond strength between resin cement and zirconia
}

\author{
XIN YUE ${ }^{1}$, XIAOYAN HOU $^{2}$, JING GAO $^{1}$, PINGPING BAO $^{3}$ and JING SHEN $^{1}$ \\ ${ }^{1}$ International Medical Center, Tianjin Stomatological Hospital, Tianjin 300041; \\ ${ }^{2}$ Department of Stomatology, Tianjin First Hospital, Tianjin 300232; ${ }^{3}$ Department of Endodontics, \\ Tianjin Stomatological Hospital, Tianjin 300041, P.R. China
}

Received March 9, 2018; Accepted December 6, 2018

DOI: $10.3892 /$ etm.2019.7382

\begin{abstract}
Yttria-stabilized tetragonal zirconia polycrystal (Y-TZP) ceramics are widely used for fixed dental prostheses and dental implants. Various primers have been introduced to enhance the bonding strength of zirconia crowns. The aim of current study was to analyze the effects of 10-methacryloyloxydecyl dihydrogen phosphate (MDP) primers on the shear bond strength between zirconia crowns and resin cement were assessed. The total sample size included 90 samples of Y-TZP ceramic surfaces. Samples in group I were abraded with aluminum oxide. Samples in groups II and III were abraded and primed with the zirconia and Z-PrimePlus MDP primers, respectively. Group IV samples did not undergo any treatment or application. Group V and VI samples did not undergo any abrasion but were primed with zirconia and Z-PrimePlus MDP primers, respectively. Prior to bonding of the resin cement, the primers were applied for $1 \mathrm{~min}$ and air-dried. Then, Clearfil SA luting self-adhesive resin cement was coated onto the zirconia crowns. The highest bond strength was achieved in group III, followed by group II, and group IV exhibited the lowest bond strength between Y-TZP ceramic and adhesive resin cement. Therefore, the application of MDP primers yielded greater bond strength between Y-TZP ceramics and adhesive resin cement, compared with the group without the use of primers.
\end{abstract}

\section{Introduction}

At present, there is a high demand for full ceramic crowns on fixed partial dentures that are completely metal free. The most commonly used ceramic core material is the yttria-stabilized tetragonal zirconia polycrystal (Y-TZP)

Correspondence to: Dr Jing Shen, International Medical Center, Tianjin Stomatological Hospital, 75 Dagu North Road, Tianjin 300041, P.R. China

E-mail: shakirahtterg@yahoo.com

Key words: 10-methacryloyloxydecyl dihydrogen phosphate primers, zirconia, bond strength, resin cement ceramic, also known the zirconia crown, due to its optimal mechanical and esthetic properties (1). These crowns are highly resistant to fracture owing to their physical properties (1). We propose that ceramics offer long-term durability, however, saliva commonly causes stress corrosion by reacting with the glass structure, further causing decomposition. Ceramic crowns are also associated with subcritical crack propagation $(2,3)$. By contrast, metal crowns and cores are entirely glass free due to their polycrystalline microstructures, and, therefore offer durability, stability and minimal risk of structural decomposition (3). Few in vitro studies have been conducted on this subject, and those conducted did not reach definitive conclusions regarding the bond strength between resin cement and zirconia. Researchers and scientists have attempted to alter and modify the superficial surface properties of zirconia by adopting numerous methodologies, including abrasion with airborne particles $(4,5)$. The use of tribochemical silica coating, a relatively new method of air abrasion, has increased $(4,5)$. This method involves the use of small $(\sim 100 \mu \mathrm{m})$ aluminum oxide particles coated with silica (4-7). When an appropriate blasting pressure is applied, silica-coated aluminum particles are embedded into the surface, which increases its sensitivity and chemical activity, resulting in the formation of silane molecules (6). These molecules react further with water to form the silanol group as well as the corresponding methoxy group (8-10). Trimethoxysilyl-propyl methacrylate (MPS) is the most commonly used silane in dental labs. Therefore, in order to optimize compatibility, we propose that resin composite materials i.e. MPS, should have one component that has been adapted in conjugation with MPS silane.

The use of chair side abrasion techniques for air abrasion is also increasing (7). These techniques facilitate the increase in surface energy and surface area, further enhancing the adhesion between resin cement and zirconia while also increasing micromechanical retention (8). Another advantage of this method is that it decreases surface tension, thereby augmenting the wettability of silane particles and improving adhesion. Following chair side airborne abrasion of the silane particles, application of primers containing 10-methacryloyloxydecyl dihydrogen phosphate (MDP) significantly enhances bonding and durability (9). MDP-based primers also function as adhesion promoters and are easy to use and apply, cost-efficient, 
and do not require specialized equipment for their application $(10,11)$.

Various primers designed to enhance the bonding strength of zirconia ceramic have been introduced (12). The use of MDP-containing primers on the zirconia surface and the use of phosphate monomers as reliable chemical agents for improving zirconia bonding have been supported previously (13). A plausible mechanism underlying this observation is the ability of phosphate monomers to form chemical bonds with the zirconia surface, resulting in polymerizable resin terminal end groups, for example MPS, which facilitate cohesive bonding to the resin cement (14).

The chemical adhesion potential of zirconia is low due to inertness of its non polar surface, which impedes its bonding with cements. However, increased availability of hydroxyl groups was observed at the implant surface of a zirconia/aluminumna no composite following treatment with a $15 \mathrm{M}$ sodium hydroxide solution. Additionally, durable bond strength may be achieved by employing acid monomers, including MDP-based primers (13-15).

The most commonly used and appropriate resin cement in prosthodontics is Panavia F2.0, which is composed of a bi-functional monomer and MDP (15). Several authors have suggested that Panavia F2.0 improves bonding between the zirconia and resin cement (15). With recent advances in these materials, the use of Y-TZP in restorative dentistry as a core material for ceramic crowns and bridges has increased. Y-TZP exhibits higher fracture toughness and strength compared with dental ceramics. (16) Despite the high fracture resistance of Y-TZP, resin cements are preferable to conventional cements such as zinc polycarboxylate cement and glass-ionomer cements for luting ceramic restorations, due to their superior retention properties, increased fracture resistance and good marginal seal (17). Various other novel techniques have been developed to increase the surface roughness of zirconia, including laser etching using erbium-doped yttrium aluminum garnet laser, $\mathrm{CO}_{2}$, and neodymium-doped yttrium aluminum garnet lasers (18).

Therefore, several methods including sandblasting, plasma spraying, silane application, tribochemical silica coating, acid etching and heat-based selective maturation etching techniques have been applied to improve the adhesiveness and shear bond strength (SBS) between zirconia and resin cement. None of these, however, have proven satisfactory or yielded the desired results, according to our analysis (19). The aim of this study was to analyze the effects of MDP-containing primers on the SBS between resin cement and zirconia.

\section{Materials and methods}

Specimen preparation. A total of 90 zirconia samples $(15 \times 10 \times 4 \mathrm{~mm})$ were prepared using a low-concentration diamond blade from pre-sintered Y-TZP blocks [97\% zirconium dioxide stabilized with $3 \%$ yttria (3M ESPE, Loughborough, UK; Table I)]. Each sample was initially ground for surface finishing and polished using 600-grit silicon carbide abrasive burs under running water. Each sample was further cleaned ultrasonically for $10 \mathrm{~min}$ under-distilled water. Then the specimens were heated at $1,300^{\circ} \mathrm{C}$ for $12 \mathrm{~h}$. Finally, the samples were embedded in clear acrylic resin blocks (ISOMET4000;
Buehler, Lake Bluff, IL, USA). For cement bonding, a single side of each block was left exposed. Surface modifications, bonding procedures, evaluation of bond strength, evaluation of failure, surface characterization and thermo dynamic calculations, were performed as previously described $(18,19)$.

Surface modifications and bonding procedure. All 90 samples were divided into six groups comprising 15 samples each according to the surface treatment used. Surface treatment of the specimens was performed by air abrasion using aluminum oxide particles (particle size, $50 \mu \mathrm{m}$; stand off distance, $25 \mathrm{~mm}$; pressure, 2.8 bar; duration, $15 \mathrm{sec}$ ). The bonding agent was Adper Scotchbond Multi-Purpose Plus Adhesive (3M ESPE).

Treatment groups. Samples in group I were air abraded with aluminum oxide. Samples in groups II and III were air abraded with aluminum oxide and treated with Yttria-stabilized zirconia primer (zirconia; Ivoclar Vivadent, Ltd., Leicester, UK) primer (group II) or Z-Prime Plus MDP primer (Ivoclar Vivadent, Ltd.; group III). The samples in group IV did not undergo any treatment or primer application. In groups V and VI, the samples did not undergo any air abrasion but were treated with zirconia primer (group V) or Z-Prime Plus MDP primer (group VI; Fig. 1).

Bonding procedure. Prior to bonding of the Clearfil SA luting self-adhesive resin cement (Kuraray Noritake Dental, Inc., Okayama, Japan), the primers were applied for $1 \mathrm{~min}$ and air-dried. Subsequently, Clearfil SA luting self-adhesive resin cement was coated onto the zirconia crowns, with plastic tubes placed in the center of the samples and resin composite filling the tube. Resin cement was light-polymerized from opposite sides for $40 \mathrm{sec}\left(\mathrm{DEMI}^{\mathrm{TM}}\right.$ light-curing unit; Kerr Corporation, Orange, CA, USA). All samples were stored in distilled water for $24 \mathrm{~h}$ and then for 14 days. Analyses were performed following the 24-h and 14-day storage periods. Universal adhesive (Clearfil Universal Bond; Kuraray Noritake Dental, Inc.) was also applied on the groups which were airabrated.

Bond strength, failure, and fracture surface evaluation. To evaluate the SBS between the Y-TZP block samples and resin cement, the bonding machine (Lloyd Universal Testing Machine; Ametek, Inc., Berwyn, PA, USA) was operated at a $0.5 \mathrm{~mm} / \mathrm{min}$ crosshead speed; the adhesive interface of each specimen was loaded into a jig of the universal testing machine (Lloyd Universal Testing Machine; Ametek, Inc.) until failure occurred. The corresponding software recorded the maximum stress $(\mathrm{MPa})$ required to produce a failure. The failure mode was observed with scanning electron microscopy (SEM; magnification, x150). An investigation of the resin bonding on the zirconia and fractured surfaces was also performed using SEM. An (SEM; S-4700 FE-SEM (Hitachi, Ltd., Tokyo, Japan) was used with accelerating voltage of $10 \mathrm{kV}$, working distance of $12 \mathrm{~mm}$ and spot size 50 . The specimens were sputter-coated with Sputter Coater SC7620 (Quorum Technologies, Ltd., Laughton, UK). The samples were fixed in $2.5 \%$ paraformaldehyde- $2.5 \%$ glutaraldehyde in cacodylate sucrose buffer $(0.1 \mathrm{M}$ cacodylate, $0.1 \mathrm{M}$ sucrose, $5 \mathrm{mM} \mathrm{CaCl}$, $5 \mathrm{mM} \mathrm{MgCl}, \mathrm{pH} 7.2$ ) overnight at $4^{\circ} \mathrm{C}$, rinsed in cacodylate 
Table I. Materials used in the present study and their composition.

\begin{tabular}{lll}
\hline Material & \multicolumn{1}{c}{ Composition } & \multicolumn{1}{c}{ Supplier } \\
\hline $\begin{array}{l}\text { Yttria-stabilized tetragonal } \\
\text { zirconia polycrystal }\end{array}$ & $\mathrm{ZrO}_{2}+\mathrm{HfO}_{2}(94.4 \mathrm{wt} \%), \mathrm{Y}_{2} \mathrm{O}_{3}(5.2 \mathrm{wt} \%)$, & 3M ESPE, Loughborough, UK \\
Zirconia primer & $\mathrm{Al}_{2} \mathrm{O}_{3}(0.2-0.5 \mathrm{wt} \%)$ & \\
& $\begin{array}{l}\text { Ethanol }(30-70 \%), \text { acetone }(30-70 \%), \\
\text { silane (MPS) }(1-10 \%)\end{array}$ & Ivoclar Vivadent Ltd., Leicester, UK \\
Clearfil SA luting & Bis-GMA, EDGAMA, MDP, barium & \\
glass, silica, sodium fluoride & Kuraray Noritake Dental, Inc., Okayama, \\
Z-PrimePlus & Ethanol $(<90 \%)$, biphenyl dimethacrylate & Japan \\
& $(<10 \%)$, HEMA $(<20 \%)$, MDP & BISCO Dental Products, Schaumburg, IL, \\
& USA
\end{tabular}

MPS, trimethoxysilylpropyl methacrylate; MDP, 10-methacryloyloxydecyl dihydrogen phosphate; Bis-GMA, bisphenol A-glycidyl methacrylate; EDGAMA, ethylene glycol dimethacrylate; HEMA, Hydroxyethylmethacrylate; wt\%, weight percent.

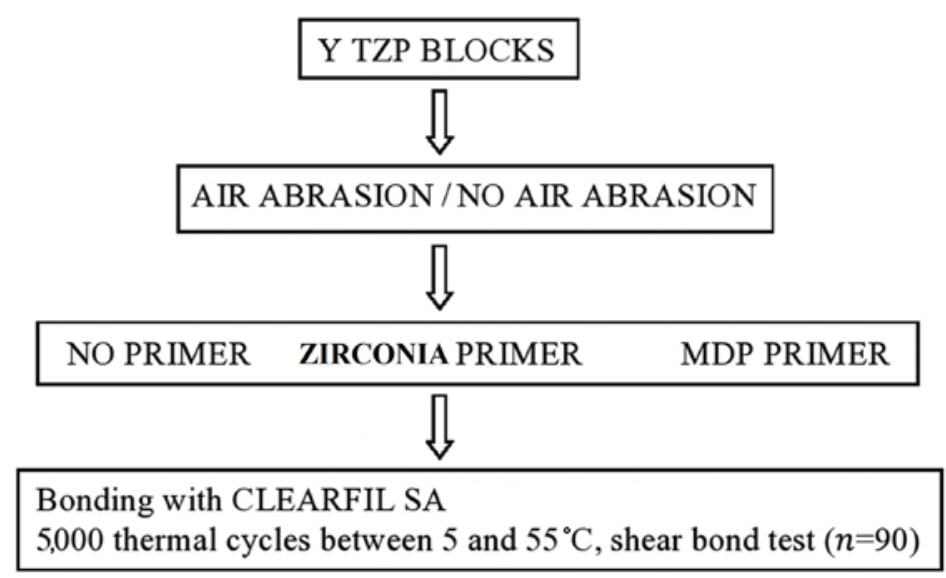

Figure 1. Flow chart of primer application. MDP, 10-methacryloyloxydecyl dihydrogen phosphate.

buffer at pH 7.2 (3 changes for $30 \mathrm{~min}$ at $4^{\circ} \mathrm{C}$ each) and then post-fixed in $1 \%$ osmium tetroxide in the same buffer for $1 \mathrm{~h}$ at $4^{\circ} \mathrm{C}$ for the analysis of the samples.

The SBS tests were performed using a universal testing machine with a $50 \mathrm{kgf}$ load cell, at a constant crosshead speed of $0.5 \mathrm{~mm} / \mathrm{min}$. The force was concentrated on the Y-TZP cement interface. The SBS $(\sigma)$ value in MP a was determined using the following equation:

$$
\sigma=\frac{P}{A}
$$

Where $P$ is the maximum load in $\mathrm{N}$ required to produce a fracture, and $A$ is the adhesive cross-sectional area, expressed as $\pi r^{2}$, where $r$ is the diameter of the bonded area divided by 2 , measured using a digital caliper (Mitutoyo Corporation, Tokyo, Japan).

The fractured surfaces were further inspected using SEM, as detailed above, at x150 magnification aided by an external light source (Leica CL5150D; Leica Microsystems, Inc., Buffalo Grove, IL, USA).

Using the grading method (20) to assess the failure mode in zirconia/cement interfaces, failure was classified as: i) Mode 1, where adhesive failure occurred between the ceramic and cement; or ii) mode 2 , where mixed failure defined as the combination of adhesive and cohesive failures occurred.
Representative specimens from each group were examined using SEM at 150x magnification.

Surface characterization of the air abraded Y-TZP surface. Fine-coated tribochemical silica with Silicatized Cojet ${ }^{\mathrm{TM}}$ sand was applied to the Y-TZP surfaces and further air abraded with aluminum oxide. Following air abrasion, the surface was sputter coated with gold particles. All collected specimens were analyzed by SEM, at 150x magnification as described above. An energy-dispersive X-ray was used for micro-analysis of the elemental distribution of alumina and silica on the sandblasted Y-TZP surfaces.

In order to observe the chemical bond between Y-TZP and MDP, the main functional adhesive composition in the current testing primers or adhesives, Y-TZP-treated MDP ethanol solution was detected using X-ray photoelectron spectroscopy (XPS; Physical Electronics, Inc., Chanhassen, MN, USA) (21).

Thermodynamic calculations. Thermodynamic calculations were performed to evaluate the stability between the zirconia and MDP primers. Several studies have modeled the MDP-bound zirconia in the tetragonal phase and analyzed the data under the standard temperature and pressure (pressure, $1 \mathrm{~atm}$; temperature, $298 \mathrm{~K}$ ) to evaluate the chemical bonds among MDP-bound zirconia, silicon oxide and MPS $(22,23)$. 
The thermodynamic calculations were performed using the Own N-layered integrated molecular orbital and molecular mechanic method in Gaussian 09 software (Gaussian, Inc., Wallingford, CT, USA).

Statistical analysis. Statistical analyses were performed using SPSS software (version 19; IBM Corp., Armonk, NY, USA). The SBS values were evaluated by one-way analysis of variance to analyze the effects of chemical surface treatments of Y-TZP. A post hoc Tukey test was applied to identify pairwise differences among the tested groups. $\mathrm{P}<0.05$ was considered to indicate a statistically significant difference.

\section{Results}

Shear bond strength testing. Differences in SBS between groups are presented in Table II. Group III (Z-PrimePlus and air abrasion) exhibited an SBS of $14.56 \pm 2.6 \mathrm{MPa}$ after $24 \mathrm{~h}$, the highest value among the groups during the first $24 \mathrm{~h}$ of storage, and $12.97 \pm 1.8 \mathrm{MPa}$ after 14 days. Significant differences were observed between group III and group IV that received no surface treatment or Z-Prime Plus primer (Table II). Group II exhibited lower SBS than group III. Surface treatment of specimens with aluminum oxide was associated with higher SBS values compared with the groups that received no surface treatment.

Air abrasion caused surface roughness with sharp edges and grooves, according to SEM. Aluminum oxide-air abraded surfaces treated with Z-Prime Plus primer had minimal fractures. Representative SEM images (magnification, x150) of Clearfil SA luting cement residue on the contact area of the Y-TZP specimens are presented in Figs. 2-4. The zirconia surfaces following cementation in group IV (control group), having received no primer treatment or air abrasion, and in group I (air abraded with aluminum oxide) are presented in Fig. 2A and B.

The zirconia surfaces following cementation in the group treated with primer and without air abrasion (Fig. 3A), the group treated with primer and air abrasion (Fig. 3B), the group treated with Z-Prime Plus MDP ceramic primer without air abrasion (Fig. 4A) and the group treated with Z-Prime Plus MDP and air abrasion (Fig. 4B) are presented.

Surface characterization of air abraded Y-TZP. The morphology of Y-TZP following different surface treatments was observed by SEM. The results of the tribochemical air-abraded process are illustrated in Figs. 5 and 6. The distribution of aluminum particles was more dense and uneven (Fig. 5A) compared with the silica particles, which were uniformly scattered, consistent in pattern, and less dense, but this difference was not marked (Fig. 5B).

The surface roughness of the surface-treated zirconia and the surface morphology are illustrated in Fig. 6A-F. The surface roughness of Y-TZP air abraded with alumina, MDP primer and tribochemical silica were not different, however, the highest value was observed in the air abraded with MDP primer group. The grooves and bridges of the groups appeared to be irregularly distributed. Generally, air abrasion combined with the use of the MDP primer caused the roughest surfaces. Remaining resin cement is presented in Fig. 6A-F. Fig. 6F presents a unique ridged appearance, with more resin cement
Table II. Shear bond strength (MPa) of samples.

\begin{tabular}{lcc}
\hline Y-ZTP samples & $\begin{array}{c}\text { Shear bond strength } \\
\text { after } 24 \mathrm{~h}\end{array}$ & $\begin{array}{c}\text { Shear bond strength } \\
\text { after 14 days }\end{array}$ \\
\hline Group I & $8.37 \pm 1.2 \mathrm{MPa}$ & $6.04 \pm 0.6 \mathrm{MPa}$ \\
Group II & $9.83 \pm 2.1 \mathrm{MPa}$ & $7.34 \pm 1.3 \mathrm{MPa}$ \\
Group III & $14.56 \pm 2.6 \mathrm{MPa}$ & $12.97 \pm 1.8 \mathrm{MPa}$ \\
Group IV & $3.97 \pm 0.9 \mathrm{MPa}$ & $1.9 \pm 0.4 \mathrm{MPa}$ \\
Group V & $6.02 \pm 1.2 \mathrm{MPa}$ & $4.91 \pm 1.8 \mathrm{MPa}$ \\
Group VI & $11.2 \pm 2.0 \mathrm{MPa}$ & $8.30 \pm 2.2 \mathrm{MPa}$ \\
\hline
\end{tabular}

Data are presented as the mean \pm standard deviation. ${ }^{a} \mathrm{P}<0.005$ vs. all other groups.

residues attached to the Y-ZTP surface in the group with a combination of air abrasion and Z-Prime Plus application.

Chemical bond characterization of primers and adhesives on $Y$-TZP. X-ray photo electron spectroscopy was used to analyze the chemical state of zirconia associated with MDP primer surfaces $(24,25)$. The chemical state of the Y-TZP-MDP surface was analyzed by XPS, and its individual peaks are presented in Fig. 7. The Zr-3d signal was divided into two peaks centered at 181 and $184 \mathrm{eV}$, in parallel with $\mathrm{Zr}-3 \mathrm{~d}_{5 / 2}$ and $\mathrm{Zr}-3 \mathrm{~d}_{3 / 2}$, respectively $(21,24)$. The $\mathrm{Zr} 3 \mathrm{~d} 5 / 2$ state indicates complete oxidation of $\mathrm{Zr} 4+$, while $\mathrm{Zr} 3 \mathrm{~d} 3 / 2$ represents spin-orbit splitting. The highest peak was observed at $\sim 532 \mathrm{eV}$. The results indicated that $\mathrm{ZrO}$ and MDP were well formed, with the highest percentage and ratio of $\mathrm{ZrO}$ (Fig. 7).

Thermodynamic calculations. The potential chemical bonds within the sandwich-like zirconia-resin cement-dentin interface were schematically illustrated in Fig. 8. Two interfaces were present: One between the resin cement and universal adhesive on the dentin surface and the other between the resin cement and universal adhesive on the Y-TZP surface. on the Y-TZP surface. Hydrolysis of the MDP and tetragonal zirconium cluster complex is expressed as follows:

$\mathrm{R}-\mathrm{OPO}_{2}-\mathrm{Zr}_{4} \mathrm{O}_{8} 2-(\mathrm{aq})+2 \mathrm{H}_{2} \mathrm{O}(\mathrm{aq})$ hydrolys is $\rightarrow$ $\mathrm{R}-\mathrm{OP}(\mathrm{OH})_{2}(\mathrm{aq})+\mathrm{Zr}_{4} \mathrm{O}_{8}(\mathrm{aq})+2 \mathrm{OH}-(\mathrm{aq})$ (21). This thermodynamic formula yielded an equilibrium constant $(\mathrm{K})$ of $4.9 \times 10^{44}$. Hydrolysis of the silica and $\mathrm{SiO}_{2}$ cluster complex is expressed as follows: $\mathrm{R}-\mathrm{SiOMe}-\mathrm{H}_{10} \mathrm{Si}_{6} \mathrm{O}_{18}(\mathrm{aq})+2 \mathrm{H}_{2} \mathrm{O}(\mathrm{aq})$ $\rightarrow \mathrm{R}-\mathrm{SiOMe}(\mathrm{OH})_{2}(\mathrm{aq})+\mathrm{H}_{12} \mathrm{Si}_{6} \mathrm{O}_{18}(\mathrm{aq})(21)$.

This thermodynamic formula yielded $\mathrm{K}=2.7 \times 10^{15}$. According to the equilibrium constants of the above formulae, the silica and $\mathrm{SiO}_{2}$ cluster complex (lower K) (21) is more stable in water than is the MDP and Y-TZP cluster complex.

Stereoscopic observations revealed adhesive failure at the interface between the zirconia ceramic and bonding agent. The fracture patterns exhibited mixed and adhesive failure following 14 days of storage of the specimens (Fig. 9).

\section{Discussion}

The untreated Y-TZP surfaces (group IV) exhibited the lowest bond strength. A high rate of adhesive failure was observed 

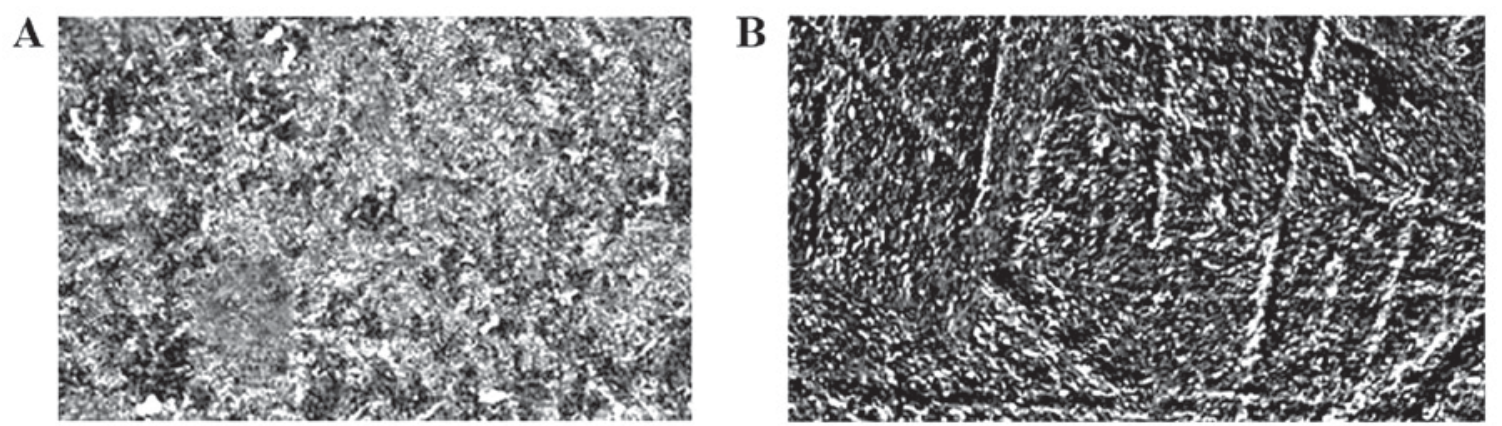

Figure 2. (A) Y-TZP ceramic specimens bonded after cementation without primer application and without air abrasion (Group IV). (B) Y-TZP ceramic specimens bonded after cementation without primer application with air abrasion (Group I). Magnification, x150. Y-TZP, yttria-stabilized tetragonal zirconia polycrystal.

A

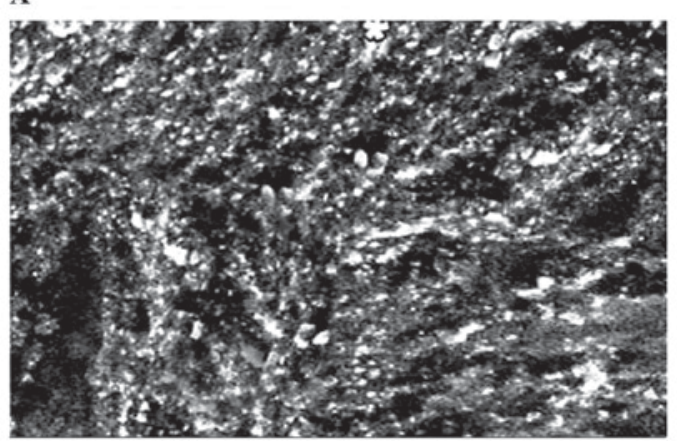

B

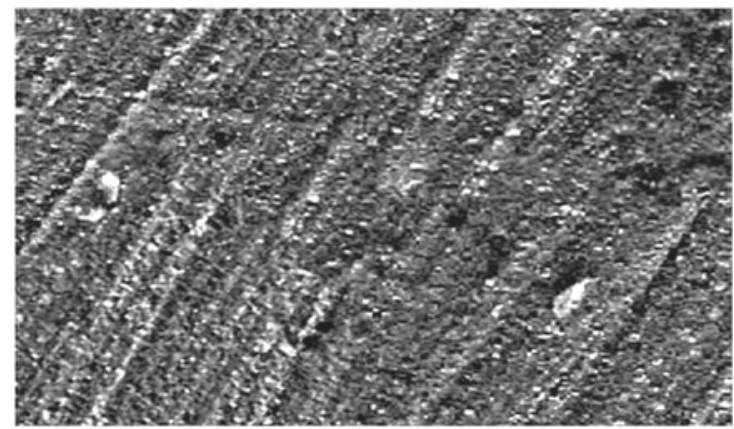

Figure 3. (A) Y-TZP ceramic specimens bonded after cementation with a primer without air abrasion (Group V). (B) Y-TZP ceramic specimens debonded after cementation with a primer and air abrasion. Y-TZP, yttria-stabilized tetragonal zirconia polycrystal (Group II). Magnification, x150.

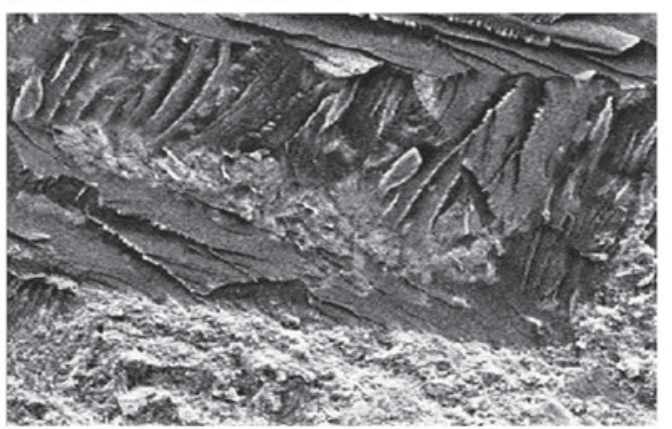

B

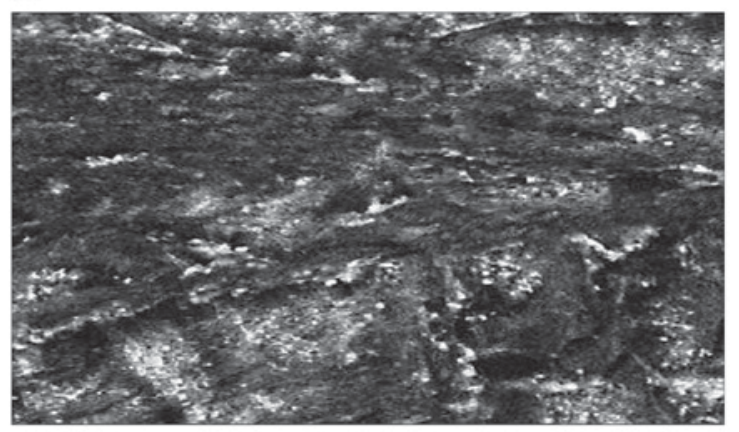

Figure 4. (A) Y-TZP ceramic specimens bonded after cementation with Z-Prime Plus ceramic primer without air abrasion (Group VI). (B) Y-TZP ceramic specimens bonded after cementation with Z-Prime Plus ceramic prime with air abrasion (Group III). Magnification, x150. Y-TZP, yttria-stabilized tetragonal zirconia polycrystal.

by SEM and the Y-TZP surface had no remnants of luting material, revealing a significantly lower SBS between the self-adhesive resin cement and untreated Y-TZP surfaces. This may be attributed to the inferior chemical bonding at the interface between the components and the interface between the MDP component of the Clearfil SA luting cement and the hydroxyl groups of the Y-TZP ceramics $(23,25)$.

Numerous studies have demonstrated low bond strength associated with the use of conventional resin cements on untreated Y-TZP ceramic surfaces (25-27). The results of the current study indicated that bond strength was significantly enhanced by treatment with MDP-based primers, as well as air abrasion.
Surface treatment without air abrasion $(50-\mu \mathrm{m}$ particles) improved bond strength, regardless of whether the zirconia under went primer pretreatment. These results are consistent with those of previous studies $(11,28)$. Treatment with air abrasion enhances surface roughness and increases surface energy, which in turn promotes resin cement flow into micro-retentions. This aids micromechanical interlocking between the resin cement and Y-TZP surface. Air abrasion may generate hydroxyl groups on the Y-TZP surfaces, increasing the reactivity of Y-TZP with phosphate monomers $(11,28)$. The results of the current study revealed that MDP-based Z-Prime Plus promoted durable and reliable 
A

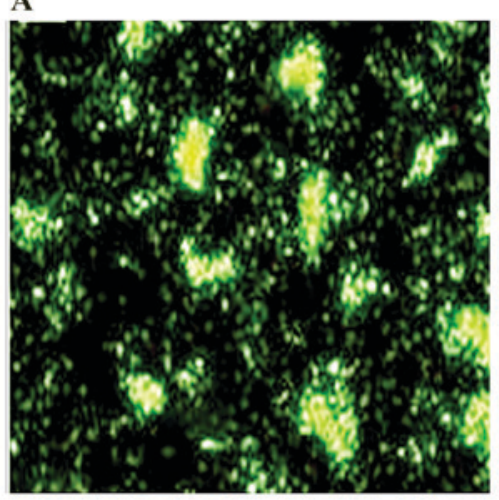

B

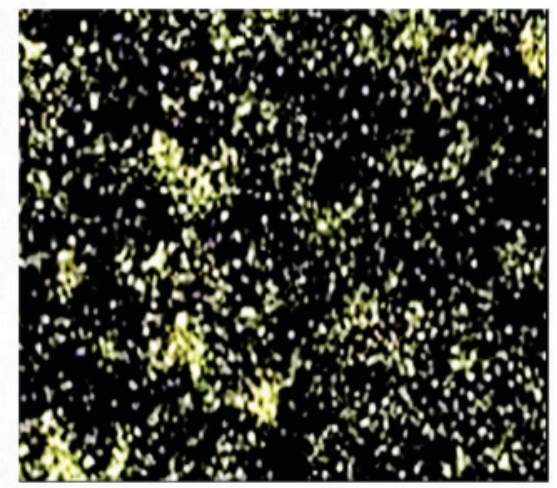

Figure 5. Surface characterization of sandblasted Y-TZP. (A) Aluminum distribution was dense and uneven. (B) Silica particles were uniformly scattered, consistent in pattern and low in density. Magnification, x150. Y-TZP, yttria-stabilized tetragonal zirconia polycrystal.
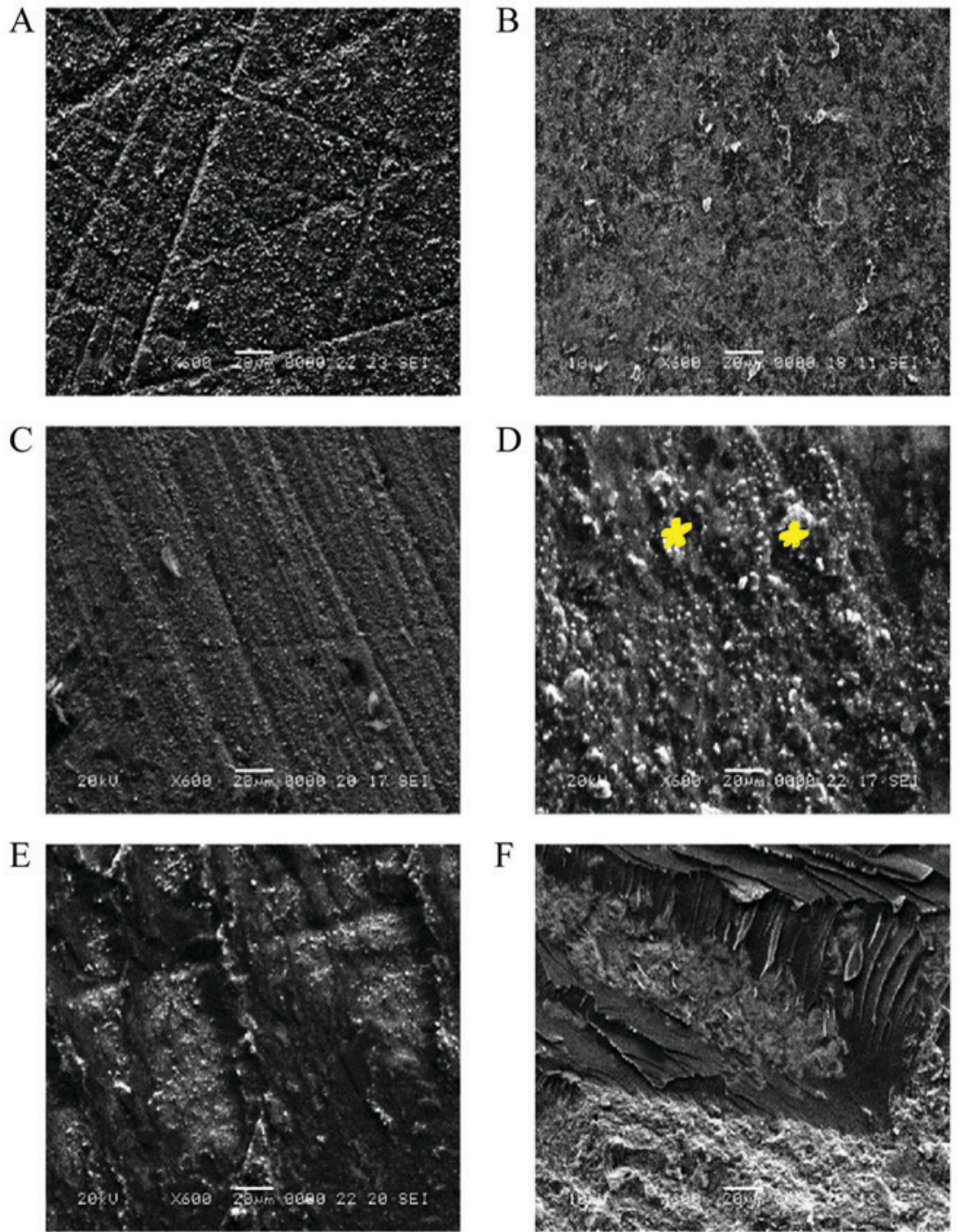

Figure 6. Representative scanning electron microscopy images of Y-TZP ceramic specimens after cementation using resin cement (magnification, $\mathrm{x} 2,000)$. (A) Polished Y-TZP. (B) Air abrasion with aluminum oxide particles (particle size, $50 \mu \mathrm{m}$ ). (C) Zirconia primer applied to polished Y-TZP. (D) Metal/zirconia primer applied to Y-TZP after air abrasion. (E) Z-Prime Plus applied to polished Y-TZP. (F) Z-Prime Plus applied to Y-TZP after air borne abrasion. The regions marked with yellow stars indicate the remaining resin cements. Y-TZP, yttria-stabilized tetragonal zirconia polycrystal.

bonding with Y-TZP $(20,28)$. Even when the self-adhesive resin cement contains MDP as one of its components, its functional monomer properties, including amount and flow, are insufficient to increase the adhesion bond to Y-ZTP without any pretreatment (22). Therefore, Y-TZP surfaces are treated with MDP-based monomers in general dental 

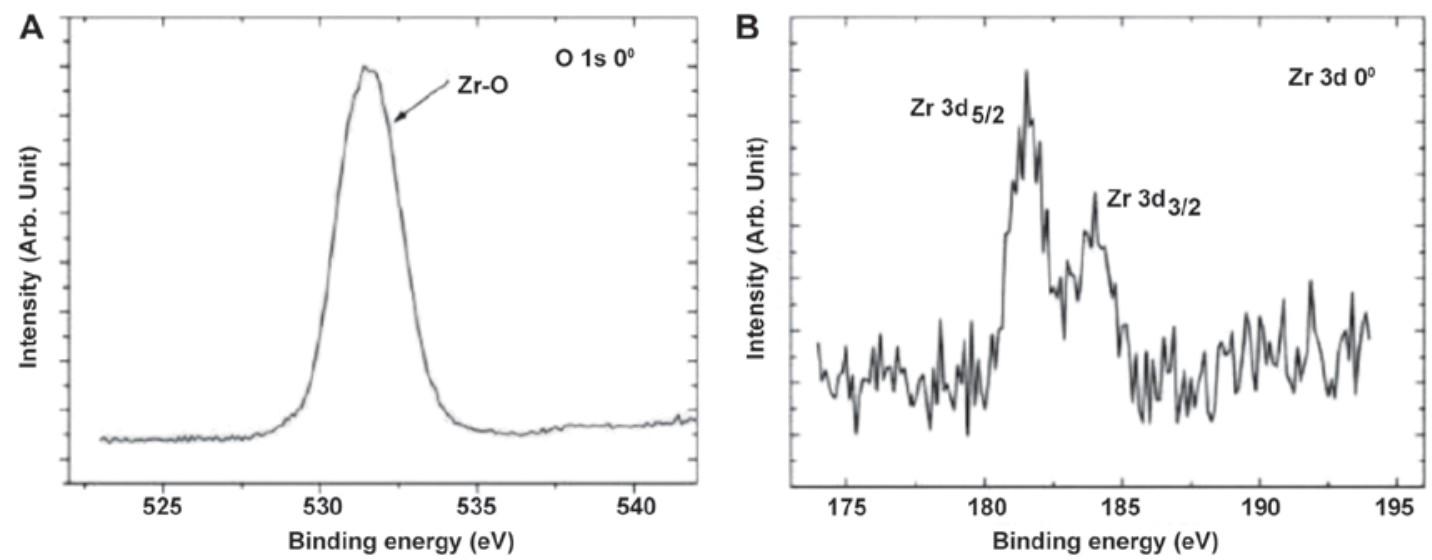

Figure 7. XPS spectra of Y-TZP surfaces. (A) ZrO-10-methacryloyloxydecyl dihydrogen phosphate was well-formed, with a high ratio of ZrO at $~ 532 \mathrm{eV}$. (B) $\mathrm{The} \mathrm{Zr} 3 \mathrm{~d} 5 / 2$ state indicates complete oxidation of $\mathrm{Zr} 4+$, while $\mathrm{Zr} 3 \mathrm{~d} 3 / 2$ represents spin-orbit splitting.

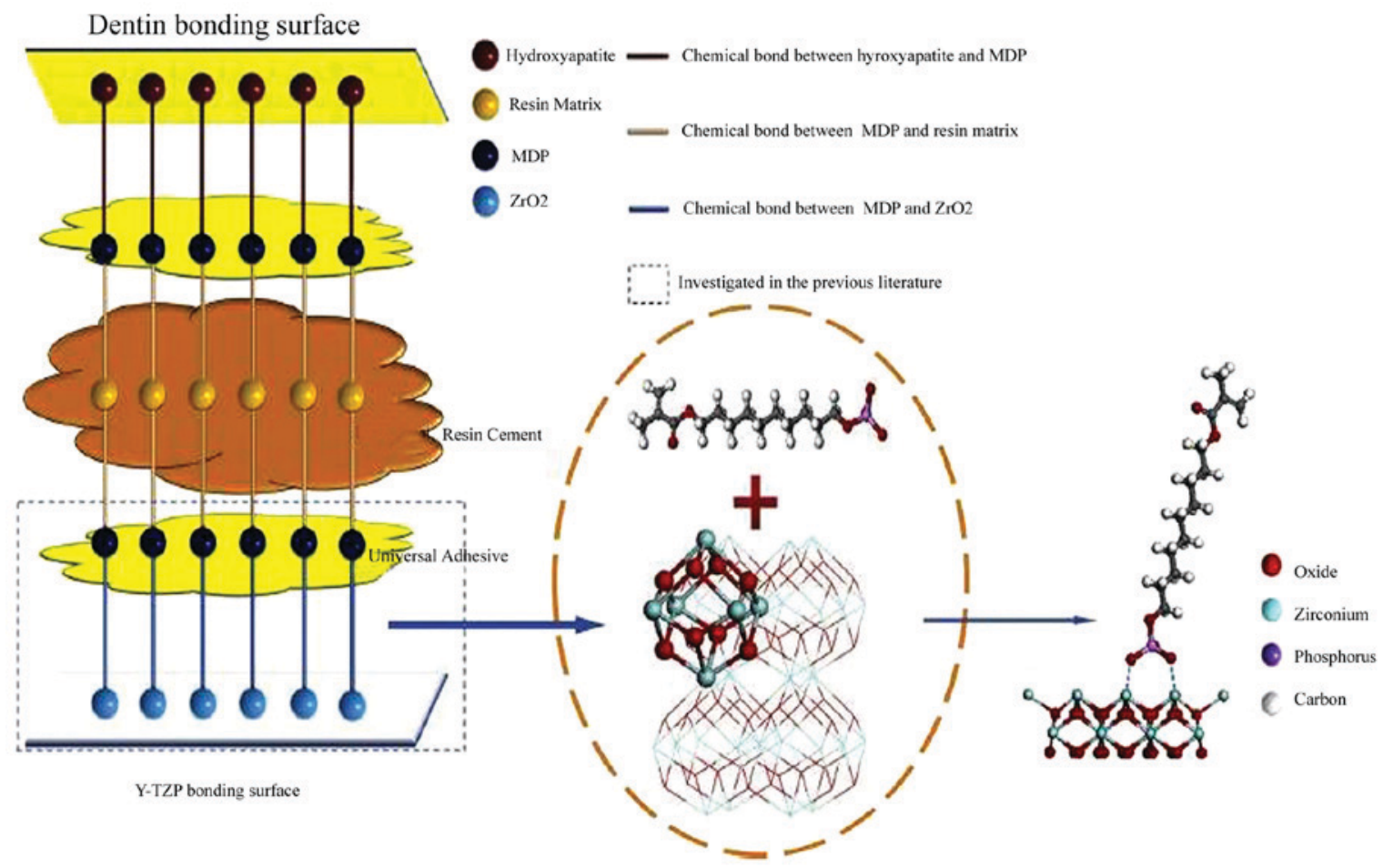

Figure 8. Chemical bonds within the sandwich-like structure consisting of two interfaces: One between the resin cement and universal adhesive on the dentin surface and the other between the resin cement and universal adhesive on the Y-TZP surface. The interface between universal adhesive and the Y-TZP bonding surface was explored previously (21). Y-TZP, yttria-stabilized tetragonal zirconia polycrystal; MDP, 10-methacryloyloxydecyl dihydrogen phosphate.

care, even if the self-adhesive resin cement contains such monomers (22). A direct bond between the phosphate ester group of the adhesive monomers and zirconia oxides may be established chemically $(23,25)$. The bifunctional ends of MDP consist of long, organic, hydrophobic chain molecules $(11,28,29)$.

In the present study, the MDP-based productZ-Prime Plus was associated with a markedly increased SBS compared with phosphonic acid-based metal/zirconia primer; this is because MDP is more effective than phosphonic acid acrylate in Y-TZP surface treatment, even when the same phosphate monomer is included in both products (19). In similar studies, MDP-based primers exhibited increased bond strength with Y-TZP, compared with other primers $(30,31)$.

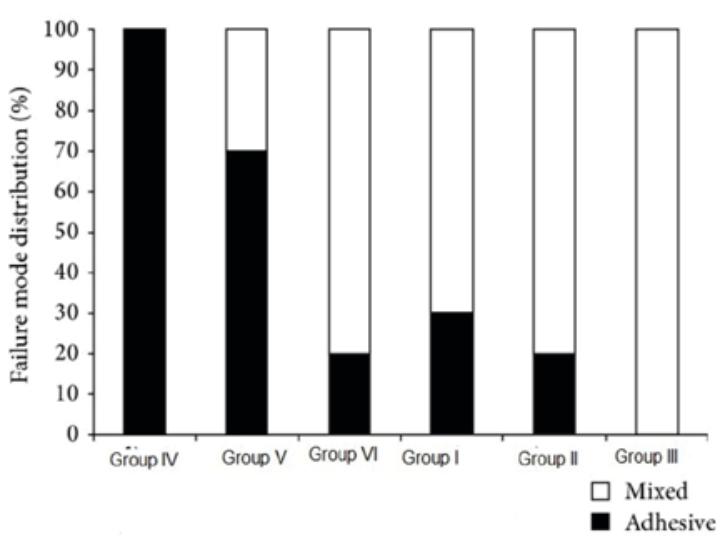

Figure 9. Distribution of failure modes. 
Adhesive failure was not limited to samples in group IV, and were observed in the groups with air abrasion due to the absence of chemical bond formation, as confirmed by SEM. The highest SBS was attained with the combination of air abrasion and the MDP-based primer. The chemical affinity in this group may have increased due to enhanced surface wettability via air abrasion and increased bond strength via treatment with MDP-based primers. In group III, all specimens exhibited a mixed fracture pattern, which may be due to the combined effects of the increased contact area with the Y-TZP ceramic surface and the improved chemical interaction.

Magne et al (32) demonstrated that treatment with Z-PrimePlus increases the SBS between zirconia ceramic and various resin-based cements. Similar results were reported by Zandparsa et al (33) and Shin et al (11), who revealed that combined application of Z-Prime Plus and air abrasion improved the bond strength between zirconia and resin cement. Furthermore, Yi et al (10) reported that the application of Z-Prime Plus following air abrasion yielded the strongest and most durable bond strength between zirconia ceramic and resin cements, followed by Monobond Plus and silane primer treatment following treatment with Cojet $^{\mathrm{TM}}$, which was consistent with the results of the current study. However, in a study by Inokoshi et al (34), pretreatment of zirconia with Clearfil ceramic primer or Monobond Plus, both containing contain silane monomers, yielded the best results, where low SBS values were observed with the use Z-Prime Plus, in contrast to the results of the present study.

Air abrasion improves bond formation between micro-roughened zirconia and resin cement $(22,23)$. In the control group IV, a high rate of failure was observed, which may be attributed to the lower bond strength compared with all other groups and poor chemical bonding between the components, as no primer had been used.

Z-Prime Plus MDP primers yielded superior results in terms of bonding to Y-TZP. The flow properties of the self-adhesive resin cement Clearfil SA are insufficient to enhance adhesion to zirconia ceramics (29). Therefore, MDP functional monomers may be required for adhesion to zirconia surfaces, even if the self-adhesive resin cement contains these monomers. This is inconsistent with previous findings (35). The phosphate ester components of the adhesive monomers are chemical agents that form direct bonds with zirconia oxides (36).

The study conducted by Nagaoka et al (29) in 2017 revealed that the combination of ${ }^{1} \mathrm{H}$ and ${ }^{31} \mathrm{P}$ magic angle spinning (MAS) nuclear magnetic resonance (NMR) and two dimensional (2D) ${ }^{1} \mathrm{H} \rightarrow{ }^{31} \mathrm{P}$ heteronuclear correlation (HETCOR) NMR enabled description of the different chemical states of the 10-MDP bonds with zirconia. These results are consistent with data presented in the current study, where, compared with all other groups, the application of MDP primers yielded the greatest bond strength between Y-TZP ceramics and adhesive resin cement (29). However, the study by Nagaoka et al (29) aimed to analyze the chemical interaction mechanism between 10-MDP and zirconia using ${ }^{1} \mathrm{H}$ and ${ }^{31} \mathrm{P}$ MASNMR and $2 \mathrm{D}^{1} \mathrm{H} \rightarrow{ }^{31} \mathrm{PHETCORNMR}$. By contrast, the current study investigated the effects of MDP-based primers on shear bond strength between resin cement and zirconia. This research may have a clinical implication in dental prosthodontic research, indicating that the application of MDP primers yielded highest bond strength between Y-TZP ceramics and adhesive resin cement, compared with other groups. By contrast, Sanohkan et al (12) concluded that the shear bond strength values between zirconia ceramic and resin composite were not significantly altered following treatment with various primers. The mode of failure for all specimens was found to be the adhesive failure at the interface between the ceramic and the bonding agent (37). The results of the current study revealed that the application of MDP primers yielded higher bond strength between Y-TZP ceramics and adhesive resin cement when compared with all other groups.

In the present study, a combination of air abrasion and treatment with MDP-based products resulted in high bond strength values and chemical affinity, due to enhanced surface wettability via air abrasion and increased bond strength via the treatment with MDP-based primers.

\section{Acknowledgements}

Not applicable.

\section{Funding}

No funding was received.

\section{Availability of data and materials}

The datasets used and/or analyzed during the current study are available from the Central Library of Tianjin Stomatological Hospital (Tianjin, China), and will be provided on reasonable request.

\section{Authors contributions}

XY compiled the project proposal, aims and objectives, and analyzed the results. XH wrote the manuscript. JG collected the data and wrote the manuscript. PB collected the data and analyzed results. JS formulated the aims and objectives, analyzed the results and submitted the manuscript.

\section{Ethics approval and consent to participate}

No patients or animals were included in this study. The research was approved by the Ethical and Research Board Committee of Medical College, International Medical Center, Tianjin Stomatological Hospital (Tianjin, China; reference no. TSH//RC/2015-12). All analyses were performed in accordance with the relevant guidelines and regulations, as instructed by the Ethical and Research Board Committee.

\section{Patient consent for publication}

Not applicable.

\section{Competing interests}

The authors declare that they have no competing interests. 


\section{References}

1. Al Amleh B, Lyons K and Swain M: Clinical trials in zirconia: A systematic review. J Oral Rehabil 37: 641-652, 2010.

2. De Sa Barbosa WF, Aguiar TR, Francescantonio MD, Cavalcanti AN, de Oliveira MT and Giannini M: Effect of water storage on bond strength of self adhesive resin cements to zirconium oxide ceramic. J Adhes Dent 15: 145-150, 2013.

3. Gonzaga CC, Cesar PF, Miranda WG, Jr and Yoshimura HN: Slow crack growth and reliability of dental ceramic. Dent Mater 27: 394-406, 2011.

4. Melo RM, Souza RO, Dursun E, Monteiro EB, Valandro LF and Bottino MA: Surface treatments of zirconia to enhance bonding durability. Oper Dent 40: 636-643, 2015.

5. Re D, Augusti D, Augusti G and Giovannetti A: Early bond strength to low pressures and blasted zirconia: Evaluation of a self-adhesive cement. Eur J Esthet Dent 7: 164-175, 2012.

6. Tanış MÇ, Akay C and Karakış D: Resin cementation of zirconia ceramics with different bonding agents. Biotechnol Biotechnol Equip 29: 363-367, 2015.

7. Murthy V, Manoharan, Balaji and Livingstone D: Effect of four surface treatment methods on the shear bond strength of resin cement to zirconia ceramics- A comparative in vitro study. J Clin Diagn Res 8: ZC65-ZC68, 2014.

8. Blatz MB, Phark JH, Ozer F, Mante FK, Saleh N, Bergler M and Sadan A: In vitro comparative bond strength of contemporary self adhesive resin cements to zirconium oxide ceramic with and without air particle abrasion. Clin Oral Investig 14: 187-192, 2010.

9. Jo EH, Huh YH, Ko KH, Park CJ and Cho LR: Effect of liners and primers on tensile bond strength between zirconia and resin-based luting agent. J Adv Prosthodont 10: 374-380, 2018.

10. Yi YA, Ahn JS, Park YJ, Jun SH, Lee IB, Cho BH, Son HH and Seo DG: The effect of sandblasting and different primers on shear bond strength between yttriatetragonal zirconia polycrystal ceramic and a self-adhesive resin cement. Oper Dent 40 : 63-71, 2015.

11. Shin YJ, Shin Y, Yi YA, Kim J, Lee IB, Cho BH, Son HH and Seo DG: Evaluation of the shear bond strength of resin cement to Y-TZP ceramic after different surface treatments. Scanning 36: 479-486, 2014

12. Sanohkan S, Kukiattrakoon B, Larpboonphol N, Sae-Yib T, Jampa T and Manoppan S: The effect of various primers on shear bond strength of zirconia ceramic and resin composite. J Conserv Dent 16: 499-502, 2013.

13. Lim MJ, Yu MK and Lee KW: The effect of continuous application of MDP-containing primer and luting resin cement on bond strength to tribochemical silica-coated Y-TZP. Restor Dent Endod 43: e19, 2018

14. Kitayama S, Nikaido T, Takahashi R, Zhu L, Ikeda M, Foxton RM, Sadr A and Tagami J: Effect of primer treatment on bonding of resin cements to zirconia ceramic. Dent Mater 26: 426-432, 2010.

15. Rohr N, Brunner S, Märtin S and Fischer J: Influence of cement type and ceramic primer on retention of polymer-infiltrated ceramic crowns to a one-piece zirconia implant. J Prosthet Dent 119: 138-145, 2018.

16. Silva LHD, Lima E, Miranda RBP, Favero SS, Lohbauer U and Cesar PF: Dental ceramics: A review of new materials and processing methods. Braz Oral Res 31 (Suppl 1): e58, 2017.

17. Thompson JY, Stoner BR, Piascik JR and Smith R: Adhesion/cementation to zirconia and other non-silicate ceramics: Where are we now. Dent Mater 27: 71-82, 2011.

18. Mirhashemi A, Sharifi N, Moharrami M and Chiniforush N: Evaluation of different types of lasers in surface conditioning of porcelains: A review article. J Lasers Med Sci 8: 101-111, 2017.

19. Ruyter EI, Vajeeston N, Knarvang T and Kvam K: A novel etching technique for surface treatment of zirconia ceramics to improve adhesion of resin-based luting cements. Acta Biomater Odontol Scand 3: 36-46, 2017.

20. Kobayashi K, Komine F, Blatz MB, Saito A, Koizumi H and Matsumura $\mathrm{H}$ : Influence of priming agents on the short-term bond strength of an indirect composite veneering material to zirconium dioxide ceramic. Quintessence Int 40: 545-555, 2009.
21. Xie H, Li Q, Zhang F, Lu Y, Tay FR, Qian M and Chen C: Comparison of resin bonding improvements to zirconia between one-bottle universal adhesives and tribochemical silica coating, which is better? Dent Mater 32: 403-411, 2016.

22. Ahn JS, Yi YA, Lee Y and Seo DG: Shear bond strength of MDP-containing self-adhesive resin cement and Y-TZP ceramics: Effect of phosphate monomer-containing primers. Biomed Res Int 2015: 389234, 2015.

23. de Souza G, Hennig D, Aggarwal A and Tam LE: The use of MDP-based materials for bonding to zirconia. J Prosthet Dent 112: 895-902, 2014.

24. Valov I, Stoychev D and Marinova T: Study of the kinetics of processes during electrochemical deposition of zirconia from nonaqueous electrolytes. Electrochimica Acta 47: 4419-4431, 2002.

25. da Silva EM, Miragaya L, Sabrosa CE and Maia LC: Stability of the bond between two resin cements and an yttria-stabilized zirconia ceramic after six months of aging in water. J Prosthet Dent 112: 568-575, 2014.

26. Lee SE, Bae JH, Choi JW, Jeon YC, Jeong CM, Yoon MJ and Huh JB: Comparative shear-bond strength of six dental self-adhesive resin cements to zirconia. Materials (Basel) 8: 3306-3315, 2015.

27. Piwowarczyk A, Lauer HC and Sorensen JA: The shear bond strength between luting cements and zirconia ceramics after two pre-treatments. Oper Dent 30: 382-388, 2005.

28. Kim JE, Kim JH, Shim JS, Roh BD and Shin Y: Effect of surface treatment on shear bond strength between resin cement and Ce-TZP/A12O3. Biomed Res Int 2016: 7576942, 2016

29. Nagaoka N, Yoshihara K, Feitosa VP, Tamada Y, Irie M, Yoshida Y, Van Meerbeek B and Hayakawa S: Chemical interaction mechanism of 10-MDP with zirconia. Sci Rep 7: 45563, 2017.

30. Cabezaa A, Gómez-Alcántaraa M, del M, Olivera-Pastora $\mathrm{P}$ Sobradosb I, Sanz J, Sanz J, Xiao B, Morris RE, Clearfield A and Arandaa MAG: From non-porous crystalline to amorphous microporous metal (IV) bisphosphonates. Microporous and Mesoporous Materials 114: 322-336, 2008.

31. Chen L, Suh BI, Brown D and Chen X: Bonding of primed zirconia ceramics: Evidence of chemical bonding and improved bond strengths. Am J Dent 25: 103-108, 2012.

32. Magne P, Paranhos MP and Burnett LH Jr: New zirconia primer improves bond strength of resin-based cements. Dent Mater 26: 345-352, 2010.

33. Zandparsa R, Talua NA, Finkelman MD and Schaus SE: An in vitro comparison of shear bond strength of zirconia to enamel using different surface treatments. J Prosthodont 23: 117-123, 2014.

34. Inokoshi M, Poitevin A, De Munck J, Minakuchi S and Van Meerbeek B: Bonding effectiveness to different chemically pre-treated dental zirconia. Clin Oral Investig 18: 1803-1812, 2014.

35. Della Bona A, Anusavice KJ and Shen C: Microtensile strength of composite bonded to hot-pressed ceramics. J Adhesive Dent 2: 305-313, 2000

36. Kern M and Thompson VP: Bonding to glass infiltrated alumina ceramic: Adhesive methods and their durability. J Prosthet Dent 73: 240-249, 1995.

37. Miragaya L, Maia LC, Sabrosa CE, de Goes MF and da Silva EM: Evaluation of self-adhesive resin cement bond strength to yttria-stabilized zirconia ceramic (Y-TZP) using four surface treatments. J Adhes Dent 13: 473-480, 2010.

This work is licensed under a Creative Commons Attribution-NonCommercial-NoDerivatives 4.0 International (CC BY-NC-ND 4.0) License. 